\title{
Local Activation Time Annotation in Atrial Electrogram Arrays Using Deconvolution
}

\author{
Bahareh Abdi ${ }^{1}$, Richard C Hendriks ${ }^{1}$, Alle-Jan van der Veen ${ }^{1}$, and Natasja MS de Groot ${ }^{2}$ \\ ${ }^{1}$ Circuit and Systems, Technical University of Delft, Delft, The Netherlands \\ ${ }^{2}$ Department of Cardiology, Erasmus University Medical Center, Rotterdam, The Netherlands
}

\begin{abstract}
An electrogram array can be modeled as a spatial convolution of transmembrane currents generated by each activated atrial cell, with an appropriate measurementfunction that depends on the cells' distance to the electrodes. Compared to electrograms, transmembrane currents suffer less from the superposition of far-field atrial activities as they are not affected by the spatial convolution. As a result, transmembrane currents represent more local information and estimation of the local activation time using the steepest deflection will be more accurate than when using fractionated electrograms. However, transmembrane current estimation from electrogram array recordings is an under-determined problem, having infinite solutions, among which the desired solution. To constrain the solution space, additional prior information can be used. As the temporal derivative of transmembrane currents are typically sparse, we use this as prior information. We use a Split Bregman method with a quick convergence rate to iteratively solve the problem. Our implementation of circulant operations in the formulation of the solution of each step, makes it possible to perform them very fast using FFTs. Using simulated electrograms, we show that the proposed approach outperforms the conventional approach of annotating the steepest deflection of electrograms as the activation time.
\end{abstract}

\section{Introduction}

Atrial fibrillation (AF) is the most common age related cardiac arrhythmia and is characterized by rapid and irregular electrical activity of the atria. Atrial electrograms play an important role in the analysis of AF and examining the level of electropathology in human tissue [1]. Wavefront activation maps, estimated from electrogram arrays, are the most common tool for interpretation and diagnosis of AF. However, the accuracy of such analyses are limited by the accuracy of the local activation time (LAT) estimation in the recorded electrograms.
In addition to the cells directly under the electrode, an electrode also measures the potential of cells in its direct surrounding. The electrode has therefore not only a local view on the cell potential, but experiences a superposition of distant atrial excitations. This can hamper the exact LAT estimation. The main goal of this paper is to introduce a fast and efficient approach to solve the deconvolution problem in order to estimate the transmembrane current and subsequently to improve LAT estimation. First we constrain the under-determined deconvolution problem by adding an appropriate constraint. This reduces the solution space. To do so, we constrain the first temporal derivative of the estimated transmembrane currents to be sparse. The problem formulation is solved using the Split Bregman method [2] with a quick convergence rate to solve the problem iteratively. Moreover, unlike traditional deconvolution approaches, the proposed approach diagonalizes the underlying operators even if some electrogram recordings are missing. This enables the fast implementation of these operations using FFTs.

\section{Electrogram Model}

We use $\phi[x, y, t]$ to denote an electrogram recorded on an assumed 2D uniform grid of modeled atrial cells where $x \in\left\{1,2, . ., r_{c}\right\}$ is the row index, $y \in\left\{1,2, . ., c_{c}\right\}$ is the column index and $t \in\{1,2, . ., T\}$ is the time sample index. As shown in literature [3], an electrogram can be modeled as a spatial convolution of per cell transmembrane currents $I[x, y, t]$ with a specific distance function $R_{0}[x, y]$, that is

$$
\phi[x, y, t]=S_{0}[x, y]\left(R_{0}[x, y] * * I[x, y, t]\right),
$$

where $* *$ is the $2 \mathrm{D}$ spatial convolution operator. In the model, we included $S_{0}[x, y]$ as a sampling function (or masking function) that equals 1 at specific coordinates where an electrode is positioned and 0 otherwise. These coordinates with no available electrograms can be the added cells at the boundaries of the electrode array or faulty electrodes recording no useful data. This is demonstrated in Figure 1. The main electrogram array of size 


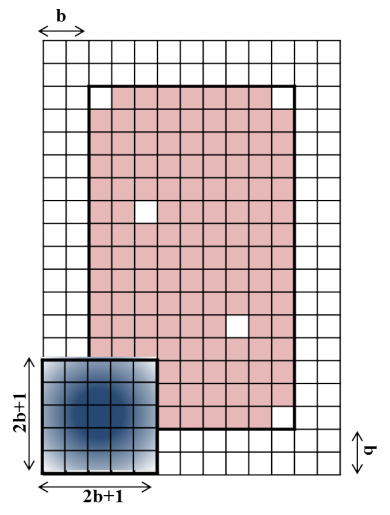

Figure 1. The 2D grid of modeled cells (in pink and white) as well as the distance function in the left bottom corner (in blue). The cells with no available electrograms are denoted by white color. These can be the cells at the extended boundary, the four electrodes in the corners that are used for different measurements, and defect electrodes.

$9 \times 15$ is shown by the light red color, the distance function $R_{0}$ of size $(2 b+1) \times(2 b+1)$ at the left bottom corner is denoted by the blue color. For a proper convolution, the boundaries of the electrode array is padded with $b$ rows and columns of cells on each side. As you can see in Figure 1, the electrogram array and the modeled cells are sampled on the same grid. However, in practice it might be more appropriate to use finer grid for cells than electrograms. We can then use $S_{0}$ to only sample cells with available electrograms as before.

\section{Methods}

\subsection{Problem formulation}

Given the model in Equation 1 we aim to deconvolve the electrograms with the distance function $R_{0}$ and estimate the trans-membrane currents $I$. However, this deconvolution problem is under-determined and has an infinite number of solutions. A typical approach to reduce the solution space, is to add prior information as a constraint. As the temporal derivatives of transmembrane currents are typically rather sparse, we use this as prior information and constrain the first temporal derivative of the estimated transmembrane currents $I^{\prime}(x, y, t)$ to have a few non-zero values, i.e., to be sparse. These sparse non-zero values represent the sharp variations in the electrogram that are also important for the further analysis of the data, because they appear when an activation wave reaches an electrode or its surrounding. Using sparsity, imposed through the $l_{1}$-norm, will help to maintain these variations. Therefore, the con- straint deconvolution problem is

$$
\begin{aligned}
\min _{\mathbf{I}} \sum_{x} \sum_{y} \sum_{t}\left|I^{\prime}[x, y, t]\right| \quad \text { s.t. } \\
\quad \phi[x, y, t]=S_{0}[x, y]\left(R_{0}[x, y] * * I[x, y, t]\right),
\end{aligned}
$$

where the objective represents the aforementioned $l_{1}$ norm. To develop a systematic approach to solve the problem formulation in Equation 2, we need to rewrite it as a system of linear equations, that is,

$$
\min _{\mathbf{i}}\left\|\mathbf{D}_{t} \mathbf{i}\right\|_{1} \quad \text { s.t. } \quad \phi=\mathbf{S} \tilde{\mathbf{R}} \mathbf{i}
$$

where vectors $\boldsymbol{\phi} \in \mathbf{R}^{N T}$ and $\mathbf{i} \in \mathbf{R}^{N T}$ are the vectorized form of all electrograms and trans-membrane currents, respectively. $N=r_{c} \times c_{c}$ the total number of modeled cells. We have also introduced three new matrices: $\mathbf{S}$ as the appropriate masking matrix of size $N \times N, \tilde{\mathbf{R}}$ as the appropriate block circulant with circulant block (BCCB) matrix that performs convolution as a matrix multiplication (with periodic boundary condition), and $\mathbf{D}_{t}$ as the first temporal derivative operator. Notice that these new matrices $\left(\mathbf{D}_{t}, \mathbf{S}\right.$ and $\tilde{\mathbf{R}}$ ) and notation are introduced to develop a systematic framework for solving the problem. While, in practice, we do not require to construct them as we can do the operations more efficiently in a transformed domain due to their special properties (see Section 3.2).

\subsection{Split Bregman algorithm}

We use the Split Bregman algorithm to reformulate Equation 3 and solve the problem formulation numerically. This algorithm converges very quickly, especially for problems with $l_{1}$-norms [2]. First we make Equation 3 into an unconstrained problem

$$
\min _{\mathbf{i}}\left\|\mathbf{D}_{t} \mathbf{i}\right\|_{1}+\frac{\lambda}{2}\|\boldsymbol{\phi}-\mathbf{S} \tilde{\mathbf{R}} \mathbf{i}\|_{2}^{2} .
$$

Next, we introduce two splitting variables $\mathbf{z}_{1}=\mathbf{D}_{t} \mathbf{i}$ and $\mathbf{z}_{2}=\tilde{\mathbf{R}} \mathbf{i}$ and two Bregman iterative parameters $\mathbf{b}_{1}$ and $\mathbf{b}_{2}$ and reformulate Equation 4 into

$$
\begin{array}{r}
\min _{\mathbf{i}, \mathbf{z}_{1}, \mathbf{z}_{2}, \mathbf{b}_{1}, \mathbf{b}_{2}}\left\|\mathbf{z}_{1}\right\|_{1}+\frac{\mu_{1}}{2}\left\|\mathbf{z}_{1}-\mathbf{D}_{t} \mathbf{i}-\mathbf{b}_{1}\right\|_{2}^{2} \\
+\frac{\lambda}{2}\left\|\boldsymbol{\phi}-\mathbf{S} \mathbf{z}_{2}\right\|_{2}^{2}+\frac{\mu_{2}}{2}\left\|\mathbf{z}_{2}-\tilde{\mathbf{R}} \mathbf{i}-\mathbf{b}_{2}\right\|_{2}^{2},
\end{array}
$$

where $\mu_{1}$ and $\mu_{2}$ are the penalty parameters. The Split Bregman algorithm solves Equation 5 by iteratively solving simple subproblems that minimize over one variable at a time. The subproblems at iteration $k+1$ and their solutions are listed below. 
1) Minimizing over $\mathrm{i}$ : In the first step, variable $\mathrm{i}$ is estimated by solving the following optimization problem,

$$
\begin{aligned}
\mathbf{i}^{k+1}= & \underset{\mathbf{i}}{\arg \min } \frac{\mu_{1}}{2}\left\|\mathbf{z}_{1}^{k}-\mathbf{D}_{t} \mathbf{i}-\mathbf{b}_{1}^{k}\right\|_{2}^{2} \\
& +\frac{\mu_{2}}{2}\left\|\mathbf{z}_{2}^{k}-\tilde{\mathbf{R}} \mathbf{i}-\mathbf{b}_{2}^{k}\right\|_{2}^{2},
\end{aligned}
$$

which can be shown to have the following solution,

$$
\begin{aligned}
\mathbf{i}^{k+1}= & {\left[\mu_{1} \mathbf{D}_{t}^{T} \mathbf{D}_{t}+\mu_{2} \tilde{\mathbf{R}}^{T} \tilde{\mathbf{R}}\right]^{-1}\left[\mu_{1} \mathbf{D}_{t}^{T}\left(\mathbf{z}_{1}^{k}-\mathbf{b}_{1}^{k}\right)\right.} \\
& \left.+\mu_{2} \tilde{\mathbf{R}}^{T}\left(\mathbf{z}_{2}^{k}-\mathbf{b}_{2}^{k}\right)\right],
\end{aligned}
$$

Since $\tilde{\mathbf{R}}^{T} \tilde{\mathbf{R}}$ and $\mathbf{D}_{t}^{T} \mathbf{D}_{t}$ are both BCCB, we can efficiently calculate $\mathbf{i}^{k+1}$ using FFTs and avoid the costly matrix inversion.

2) Minimizing over $\mathrm{z}_{1}$ : In the second step $\mathrm{z}_{1}$ is estimated by solving the following optimization problem,

$$
\mathbf{z}_{1}^{k+1}=\underset{\mathbf{z}_{1}}{\arg \min } \quad\left\|\mathbf{z}_{1}\right\|_{1}+\frac{\mu_{1}}{2}\left\|\mathbf{z}_{1}-\mathbf{D}_{t} \mathbf{i}^{k+1}-\mathbf{b}_{1}^{k}\right\|_{2}^{2},
$$

which is given by [2]

$$
\mathbf{z}_{1}^{k+1}=\operatorname{shrink}\left(\mathbf{D}_{t} \mathbf{i}^{k+1}+\mathbf{b}_{1}^{k}, \frac{1}{\mu_{1}}\right),
$$

where $\operatorname{shrink}(\alpha, \beta)=\frac{\alpha}{|\alpha|} \max (\alpha-\beta, 0)$ is the shrinkage operator.

3) Minimizing over $z_{2}$ : In the third step $z_{2}$ is estimated by solving the following equation

$$
\mathbf{z}_{2}^{k+1}=\underset{\mathbf{z}_{2}}{\arg \min } \quad \frac{\lambda}{2}\left\|\boldsymbol{\phi}-\mathbf{S z}_{2}\right\|_{2}^{2}+\frac{\mu_{2}}{2}\left\|\mathbf{z}_{2}-\tilde{\mathbf{R}} \mathbf{i}^{k+1}-\mathbf{b}_{2}^{k}\right\|_{2}^{2} .
$$

The solution to this equation is

$$
\mathbf{z}_{2}^{k+1}=\left[\lambda \mathbf{S}^{T} \mathbf{S}+\mu_{2} \mathbf{I}\right]^{-1}\left[\lambda \mathbf{S}^{T} \boldsymbol{\phi}+\mu_{2}\left(\tilde{\mathbf{R}} \mathbf{i}^{k+1}+\mathbf{b}_{2}^{k}\right)\right] .
$$

where matrix $\mathbf{S}^{T} \mathbf{S}+\mu_{2} \mathbf{I}$ is diagonal and can be easily inverted.

4) updating $b_{1}$ and $b_{2}$ : Finally, $b_{1}$ and $b_{2}$ are updated as

$$
\begin{gathered}
\mathbf{b}_{1}^{k+1}=\mathbf{b}_{1}^{k}-\mathbf{z}_{1}^{k+1}+\mathbf{D}_{t} \mathbf{i}^{k+1} . \\
\mathbf{b}_{2}^{k+1}=\mathbf{b}_{2}^{k}-\mathbf{z}_{2}^{k+1}+\tilde{\mathbf{R}} \mathbf{i}^{k+1} .
\end{gathered}
$$

\section{Results}

We use simulated two-dimensional tissues to generate electrograms and to compare the performance of our proposed method in LAT estimation, denoted by $\mathrm{SD}_{\mathrm{tmc}}$, with the conventional method, denoted by $\mathrm{SD}_{\mathrm{egm}}$. We use zones (a)

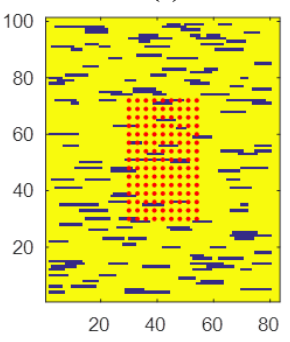

(c)
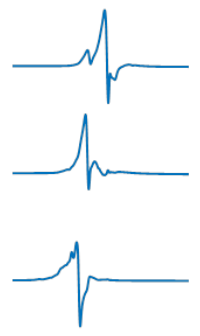

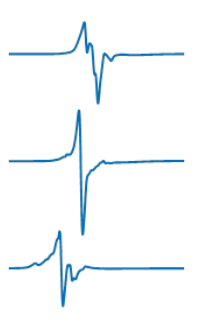

(b)
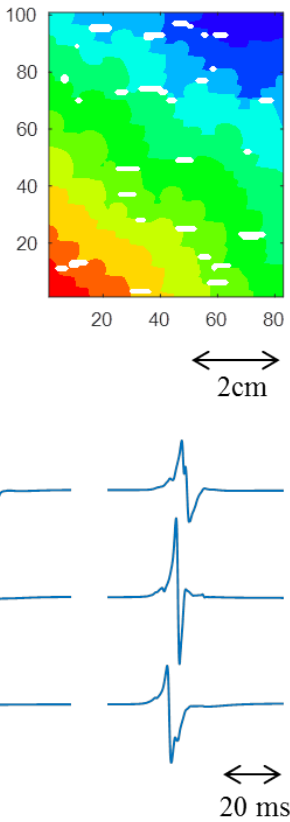

Figure 2. (a) The conductivity map of a simulated tissue, the red dots show the electrode array location. (b) The resulting activation map. (c) 9 representative simulated electrograms.

of slow conduction in tissue, introduced in [4], as a mechanism for generating fractionated electrograms. Figure 2 shows an example of the tissue's conductivity map with randomly distributed zones of slow conduction. The red dots show the location of electrodes on the tissue. The temporal resolution of electrogram recording is $0.2 \mathrm{~ms}$. The cell-to-cell distance on the tissue is $0.66 \mathrm{~mm}$, while the electrodes are sampled on a coarser grid where the electrode-to-electrode distance is $2 \mathrm{~mm}$. This makes the ratio of available electrograms to the total number of cells in the recording area 1 to 9 . We then use $\mathbf{S}_{0}$ to set the value of cells with no available electrograms to zero. In total we simulated 10 tissues and generate 1350 electrograms including 51 fractionated electrograms (electrograms with more than one distinctive descents) .

The proposed deconvolution algorithm (from Section 3) was used to estimate the transmembrane current. First, the simulated electrogram arrays were normalized to have a maximum amplitude of 1 . Vector $\mathbf{i}$ was initialized with the zero padded electrogram array. Parameters $\lambda=7 \times$ $10^{-6}, \mu_{1}=0.01$, and $\mu_{2}=0.01$ were heuristically adjusted based on visual inspection of the estimated transmembrane current estimation and a small LAT estimation error. For faster convergence, we also update $\mu_{1}$ and $\mu_{2}$ in each iteration, based on the approach proposed in [5]. The algorithm stops after 100 iterations. 


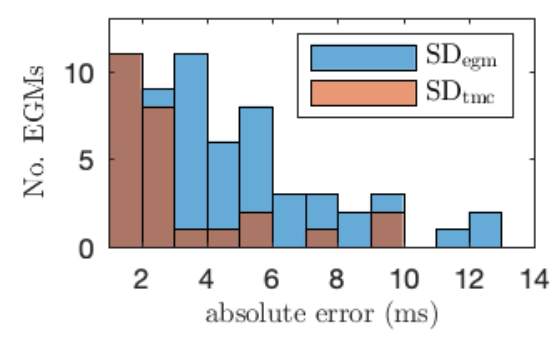

Figure 3. The histogram of absolute errors (larger than 1 ms) in LAT estimation using $\mathrm{SD}_{\text {egm }}$ and $\mathrm{SD}_{\mathrm{tmc}}$ on simulated fractionated electrograms.

Finally $\mathrm{SD}_{\mathrm{tmc}}$ and $\mathrm{SD}_{\mathrm{egm}}$ were used to estimate the LATs. $\mathrm{SD}_{\mathrm{tmc}}$ annotates the timing of the steepest descent of the estimated trans-membrane currents as the LAT, while $\mathrm{SD}_{\text {egm }}$ annotates the timing of the steepest descent of the electrograms [6]. Figure 3 shows the histogram of the absolute error between the ground truth LAT and the estimated activation time using $\mathrm{SD}_{\text {egm }}$ and $\mathrm{SD}_{\mathrm{tmc}}$ of all electrograms with absolute errors larger than $1 \mathrm{~ms}$. As can be seen, the reference algorithm $\mathrm{SD}_{\text {egm }}$ produces much larger errors (up to $13 \mathrm{~ms}$ ). These large errors usually belong to areas with a large delay and abrupt change in LAT which might be an indicator of electropathology. On the other hand, the $\mathrm{SD}_{\text {egm }}$ ignores these large delays and provides a more regular, but less correct, map.

\section{Conclusions}

A fast approach for estimation of transmembrane currents from an electrogram array through a constraint deconvolution problem, was proposed. The approach uses $l_{1}$-norm to penalize solutions with a non-sparse temporal gradient. The timing of the steepest descent in the resulting transmembrane currents (instead of the electrograms) can then be used as the LATs. We showed, by using simulated data, that the estimated trans-membrane current can be used for a better estimation of LATs.

\section{Acknowledgments}

This research has been made possible by the Dutch Heart Foundation and the Netherlands Organization for Scientific Research (NWO), as part of their joint strategic research program: "Earlier recognition of cardiovascular diseases". This project is partially financed by the PPP Allowance made available by Top Sector Life Sciences \& Health to the Dutch Heart Foundation to stimulate publicprivate partnerships.

\section{References}

[1] de Groot NM, Houben RP, Smeets JL, Boersma E, Schotten U, Schalij MJ, Crijns H, Allessie MA. Electropathological substrate of longstanding persistent atrial fibrillation in patients with structural heart disease: epicardial breakthrough. Circulation 2010;122:1674-1682.

[2] Goldstein T, Osher S. The split bregman method for 11regularized problems. SIAM journal on imaging sciences 2009;2:323-343.

[3] Chouvarda I, Maglaveras N, de Bakker JM, van Capelle FJ, Pappas C. Deconvolution and wavelet-based methods for membrane current estimation from simulated fractionated electrograms. IEEE transactions on biomedical engineering 2001;48:294-301.

[4] Jacquemet V, Henriquez CS. Genesis of complex fractionated atrial electrograms in zones of slow conduction: a computer model of microfibrosis. Heart rhythm 2009;6:803-810.

[5] Simoes M, Almeida LB, Bioucas-Dias J, Chanussot J. A framework for fast image deconvolution with incomplete observations. IEEE transactions on image processing 2016; 25:5266-5280.

[6] Spach MS, Kootsey JM. Relating the sodium current and conductance to the shape of transmembrane and extracellular potentials by simulation: effects of propagation boundaries. IEEE transactions on biomedical engineering 1985;10:743755.

Address for correspondence:

Bahareh Abdi

Faculty of Electrical Engineering, Mathematics and Computer Science, Technical University of Delft, 2628 CD Delft, The Netherlands.

b.abdikivanani@tudelft.nl 